\title{
MAGNETIC PROPERTIES OF FERROFLUID WITH COBALT FERRITE PARTICLES
}

\author{
P. DidukH ${ }^{a}$, A. Ślawska-WANieWska ${ }^{a}$, J.M. GReneche $^{b}$ \\ AND P.C. FANNIN ${ }^{c}$ \\ ${ }^{a}$ Institute of Physics, Polish Academy of Sciences \\ Al. Lotników 32/46, 02-668 Warsaw, Poland \\ ${ }^{b}$ Laboratoire de Physique, UPRESA CNRS 6087, Université du Maine \\ 72085 Le Mans, France \\ ${ }^{c}$ Department of Electronic \& Electrical Engineering, Trinity College \\ Dublin 2, Ireland
}

Low temperature Mössbauer investigations of a diluted magnetic fluid containing ultrafine $\mathrm{CoFe}_{2} \mathrm{O}_{4}$ particles are presented. The thermal evolution of cobalt ferrite particles from the magnetically ordered state to the superparamagnetic state with the blocking temperature at about $80 \mathrm{~K}$, is shown.

PACS numbers: $75.50 . \mathrm{Mm}, 76.80 .+\mathrm{y}, 61.18 . \mathrm{Fs}$

\section{Introduction}

A ferrofluid is a stable colloidal suspension of nano-sized magnetic particles dispersed in a carrier liquid. At sufficiently low temperatures, frozen ferrofluids exhibit a spin-glass type disordered structure with the frustration caused by the competing dipole-dipole interactions of randomly oriented grains [1]. At elevated temperatures the magnetic behaviour of ferrofluids is dominated by fast superparamagnetic fluctuations of the particles magnetic moments [2-4]. It is known that the magnetic properties of small particles differ from those of the corresponding bulk materials. Besides the relaxation effects, the most important factors responsible for these differences are: (i) the symmetry restriction at the particle surface, and (ii) high chemical reactivity of the surface atoms. In a ferrofluid the magnetic properties of an assembly of ultrafine particles can be changed and controlled by the surfactant used to coat the particles; the surfactant can prevent intergrain interactions but can also considerably enhance the non-collinear spin structures in the surface layer [2].

The present work was motivated by the desire to study these various surface effects. The cobalt ferrite particles were chosen since they are available in a powder form either as particles covered by the surfactant molecules or in a form of ferrofluid in which the particles are in a non-magnetic liquid. Moreover, the structure and magnetic properties of the bulk $\mathrm{CoFe}_{2} \mathrm{O}_{4}$ crystals are well known 
and can be used as reference data for the interpretation of experimental results obtained for small particles [5].

In this paper the low temperature Mössbauer investigations of a cobalt ferrite ferrofluid are presented. This method was applied since not only it can distinguish different local atomic surroundings of the $\mathrm{Fe}$ ions but is also particularly sensitive to the relaxation phenomena which are responsible for the magnetic properties of single domain particles.

\section{Experimental details}

The investigated ferrofluid consisted of a suspension of ultrafine cobalt ferrite particles in an isopar-M (a commercial hydrocarbon) carrier liquid. To prevent aggregation between the particles, they were coated by a surfactant (oleic acid). Moreover, the studied ferrofluid was in a dilute state, thus the interactions between the particles were negligible. It is known that in the bulk form the cobalt ferrite, described by the formula $\mathrm{CoFe}_{2} \mathrm{O}_{4}$, is an inverse spinel in which the $\mathrm{Co}^{2+}$ ions occupy only the octahedral (B) sites whereas the $\mathrm{Fe}^{3+}$ ions are divided between the tetrahedral (A) and octahedral (B) sites. The octahedrally coordinated $\mathrm{Co}^{2+}$ and $\mathrm{Fe}^{3+}$ ions are disordered since they occupy the $\mathrm{B}$ sites randomly.

The cobalt ferrite ferrofluid was studied by the Mössbauer spectrometry using a conventional constant acceleration spectrometer with a ${ }^{57} \mathrm{Co}(\mathrm{Rh})$ source over the temperature range $4.2-150 \mathrm{~K}$.

\section{Results and discussion}

The representative Mössbauer spectra obtained for selected temperatures are shown in Fig. 1. In the first approach the spectra were decomposed into a magnetic component with a discrete distribution of hyperfine fields and a paramagnetic doublet. The fitted curves are also presented in Fig. 1. The zero-field spectrum measured at $4 \mathrm{~K}$ of a frozen sample is magnetically split and contains a sextet with relatively sharp, well-resolved lines, indicating that all the $\mathrm{Fe}$-atoms are in magnetically ordered state. At this temperature the Mössbauer spectrum is only affected by the collective magnetic excitations caused by the fluctuations of magnetic moment near one easy axis of the magnetically ordered particle. The spectrum is composed of two main magnetic contributions, corresponding to two different Fe-sites in the spinel crystal lattice. However, these magnetic subspectra cannot be fitted with the simple Lorentzian profile of the lines as each of them contains a few hyperfine sextets.

The spectrum obtained at $60 \mathrm{~K}$ exhibits a rather complex hyperfine structure in which a magnetically split sextet with well defined maxima is superimposed on a central quadrupole doublet. This indicates the onset of fast superparamagnetic relaxations. With increasing temperature a fast reduction of the magnetically split component and simultaneous increase in the quadrupole doublet is observed. The distribution of the hyperfine fields obtained for the magnetic component is very broad and has a bimodal shape. At $150 \mathrm{~K}$ the magnetic sextet collapses completely, showing that all the particles in the ferrofluid investigated are above their blocking temperature. Figure 2 shows that the paramagnetic doublet observed at 


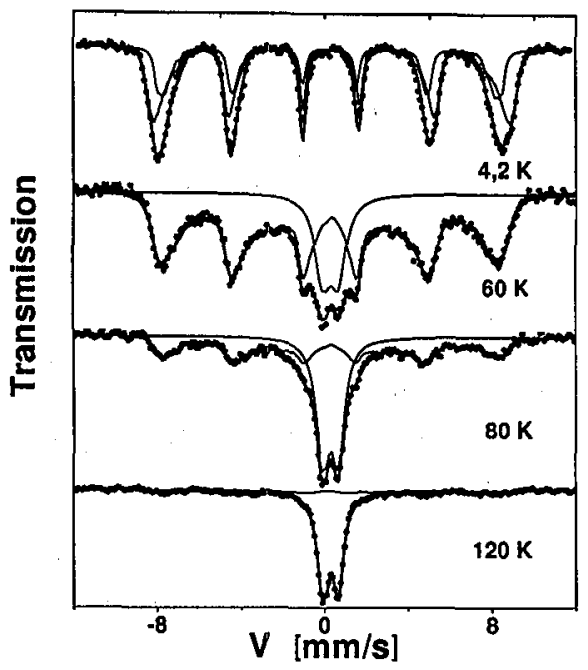

Fig. 1. Mössbauer spectra of cobalt ferrite ferrofluid at selected temperatures.

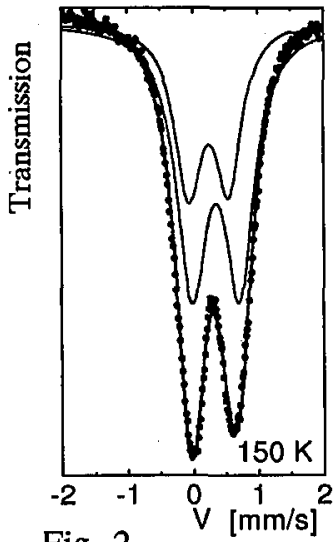

Fig. 2

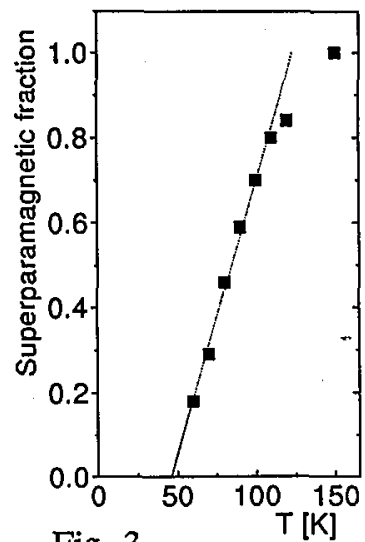

Fig. 3

Fig. 2. Mössbauer spectrum of $\mathrm{CoFe}_{2} \mathrm{O}_{4}$ ferrofluid above the blocking temperature.

Fig. 3. Thermal evolution of the superparamagnetic fraction of $\mathrm{CoFe}_{2} \mathrm{O}_{4}$ ferrofluid.

$150 \mathrm{~K}$ exhibits an asymmetry and can be decomposed a priori into two symmetrical quadrupole contributions originating from the tetrahedrally and octahedrally coordinated $\mathrm{Fe}$-ions.

The blocking temperature of the ultrafine magnetic particles measured by the Mössbauer spectrometry is usually defined as the temperature at which the magnetically split and non-split components represent $50 \%$ each of the spectral area. For the ferrofluid studied the blocking temperature can be estimated from the thermal dependence of the relative area of the superparamagnetic fraction. The corresponding results, obtained in the course of the fitting procedure of the measured spectra, are shown in Fig. 3 and reflect the distribution of the particle sizes. The blocking temperature of the cobalt ferrite particles is $T_{\mathrm{B}} \approx 80 \mathrm{~K}$ 
which roughly corresponds to a mean particle diameter of about $5.6 \mathrm{~nm}$. Figure 3 shows that for temperatures higher than $100 \mathrm{~K}$ the relative fraction of the superparamagnetic contribution is displaced from the linear behavior indicating that small aggregations of the cobalt ferrite particles in the ferrofluid studied cannot be excluded.

The Mössbauer spectra of the cobalt ferrite particles reveal two main nonequivalent Fe sites in the spinel structure. The broadening of the spectral lines as well as the wide and overlapping distributions of the hyperfine fields are observed and can be explained by: (1) the presence of the $\mathrm{Co}^{2+}$ ions in the octahedral sites which can be a source of the large local anisotropy, (2) the random arrangement of the octahedrally coordinated $\mathrm{Fe}^{3+}$ ions, (3) the surface effects connected, on the one hand, with the small particle diameters and consequently large surface to volume ratio and, on the other hand, with the surfactant molecules which are known as a source of pinning centers of the surface spins and enhance the non-collinear spin structure in the surface layer of the particles [2] as well as (4) the size-dependent reduction of the hyperfine field due to the distribution of the particle sizes. The spectra measured at different temperatures reflect the thermal evolution of the cobalt ferrite particles from the magnetically ordered state to the superparamagnetic behavior with the mean blocking temperature at about $80 \mathrm{~K}$.

A better refinement of both magnetic and quadrupole component into the independent tetrahedral and octahedral contributions is expected after performing high field Mössbauer experiments.

\section{References}

[1] D. Eberbeck, H. Ahlers, J. Magn. Magn. Mater. 192, 148 (1999).

[2] Mössbauer Spectroscopy Applied to Inorganic Chemistry, Ed. G.J. Long, Vol. 2, Plenum Press, New York 1984, p. 89.

[3] C. Johanson, M. Hanson, M.S. Pedersen, S. Morup, J. Magn. Magn. Mater. 173, 5 (1997).

[4] E. Tronc, J.P. Jolivet, Mater. Sci. Forum 235-238, 659 (1997).

[5] K.J. Davies, S. Wells, S.W. Charles, K. O'Grady, M. El Hilo, T. Meaz, S. Morup, J. Magn. Magn. Mater. 149, 14 (1995). 